\title{
Relationship between Behavioral Synchrony and Physical Loads of Care Worker and Care Recipient
}

\author{
Syogo Ogata (syougoaporo@docomo.ne.jp)1, Yutaka Fukui (fukui@engcenter.osakafu-u.ac.jp)², \\ Tsuneo Kawano (kawano@mec.setsunan.ac.jp) ${ }^{1}$
}

${ }^{1}$ Graduate School of Science and Engineering, Setsunan University, Neyagawa, Osaka, Japan, 572-8508

${ }^{2}$ College of Engineering, Osaka Prefecture University, Sakai, Osaka, Japan, 599-8531

\begin{abstract}
Tacit knowledge such as "proficient skills" and "knacks" in nursing care skills seems not to be applied by care worker alone but by the interaction between care worker and care recipient. The purpose of this study is to examine the relationship between their interaction and physical loads. And it is also to reveal the relationship of cerebral activities between care worker and care recipient. Experiments of the care operations were carried out in the care worker-recipient pairs. The body motions, muscle loads, and cerebral activities of the care worker and care recipient were measured using two sets of motion capture, EMG device, and near-infrared spectroscopy (NIRS), respectively. Experimental results indicated that the muscle loads of care worker decreased when the care recipient acted a cooperative attitude. Furthermore, in that case the cerebral activities of the care recipient were synchronized with those of the care worker.
\end{abstract}

Keywords: Care operation, Behavioral synchrony, Physical load, Motion capture, EMG, NIRS

\section{Introduction}

Musculoskeletal disorders are a widespread affliction in the nursing profession at health care institution. Nursing proficient skills are supposed to make the disorders reduce and also to lead to tender care for the care recipients. Tacit knowledge such as "proficient skills" and "knacks" in nursing care skills seems not to be applied by care worker alone but by the interaction between care worker and care recipient.

The purpose of this study is to examine the relationship between their interaction and physical loads. And it is also to reveal the relationship of cerebral activities between care worker and care recipient.

In this study care operations in which the care worker moves the care recipient from supine position to sitting position on the bed are adopted as nursing care skills and experiments of the care operations are carried out in the care worker-recipient pairs. Based on the result the relationship between behavioral synchrony and physical loads is discussed.

\section{Method}

As an exploratory experiment participants were two males as shown in Fig.1. One was for care worker (23 yrs., $176 \mathrm{~cm}$ height, $78 \mathrm{~kg}$ weight). Another was for care recipient (23 yrs., $170 \mathrm{~cm}$ height, $65 \mathrm{~kg}$ weight). The authors explained ethical considerations to the participants and obtained informed consent before the experiment.

Care operation adopted in this study was moving the

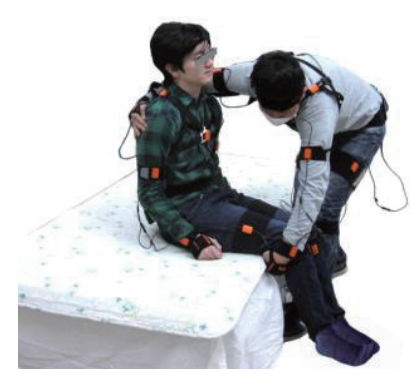

(a) Capturing care motions and measuring EMG

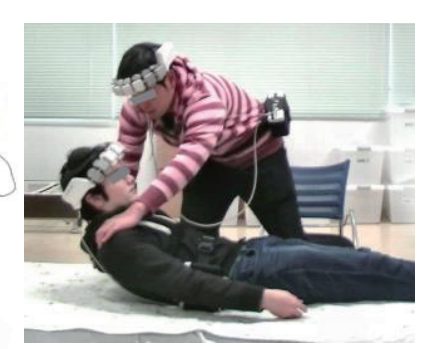

(b) Measuring brain activities by NIRS
Figure 1. Experiments of care motions 
care recipient from supine position to sitting. In the experiments the care recipient was asked to give two kinds of performances. One was to act a cooperative attitude to the care worker on purpose, and the other was to act an uncooperative one. The care operations based on their performances were repeated three times each.

The care motions, muscle loads, and cerebral activities of the care worker and care recipient were measured using two sets of motion capture (MVN, Xsens), EMG device (SYNA ACT MT-11, NEC), and near-infrared spectroscopy (NIRS) (Wearable Optical Topography, WOT-100, HITACHI), respectively.

\section{Results}

The body motions between care worker and care recipient were synchronized in the case of cooperative motions. Figure 2 shows the temporal changes of EMG during the cooperative care operations. The care worker and care recipient are extremely similar. On the other hand, the uncooperative result showed little correlation. Figure 3 shows the comparison of muscle loads between cooperative and uncooperative operation. It is recognized that the muscle loads become significantly high in the case of uncooperative operation.

Figure 4 shows the temporal changes in oxygenated and deoxygenated hemoglobin concentration at prefrontal cortices of two participants. In the case of cooperative operation, the temporal changes of $[\mathrm{Oxy}-\mathrm{Hb}]$ and [Deoxy-Hb] of two participants show a strong correlation.

\section{Conclusion}

This study was aimed to investigate the behavioral synchrony and physical loads of care worker and care recipient. Main remarks are as follows:

(1) In the cooperative care operation, the body motions between care worker and care recipient were synchronized. The changes of EMG and brain activities are also synchronized.

(2) It is suggested that muscle loads become large when the temporal changes of EMG of two participants are not synchronized.

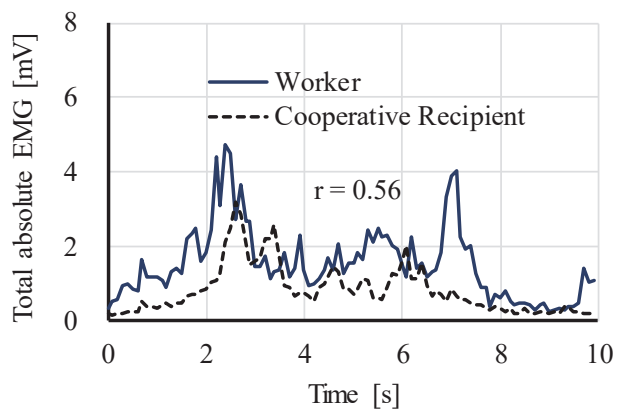

Figure 2. Envelope changes in total absolute EMG of four muscles when cooperative care operation

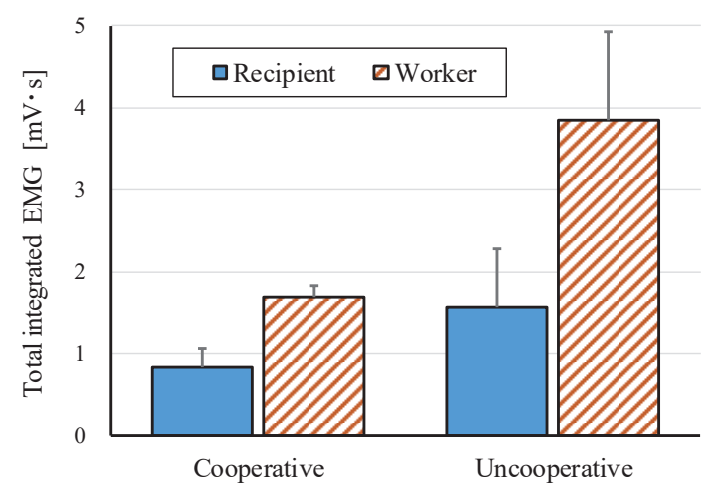

Figure 3. Comparison of muscle loads between cooperative and uncooperative operation

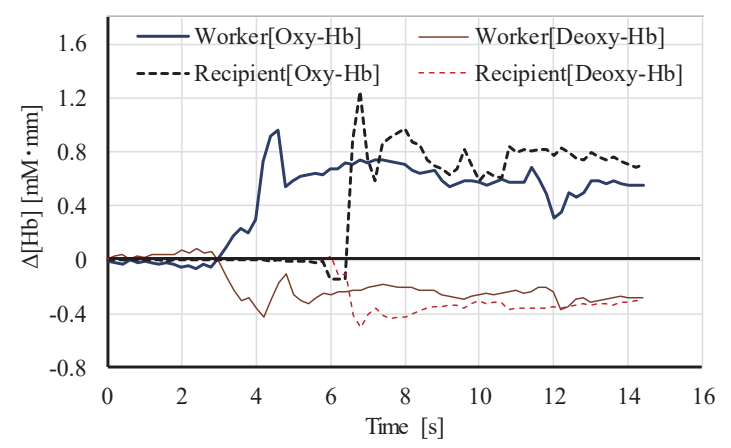

Figure 4. The temporal changes in hemoglobin concentration in the case of cooperative operation.

(3) Muscle loads seem to become large when the body motions of two participants are not synchronized.

(4) In the cooperative care operation, the cerebral activities of the care recipient were synchronized with those of the care worker.

\section{Acknowledgements}

This work was supported by a JSPS Grant-in-Aid for Scientific Research (B) (Grant-\#16H05571). 